plutôt que les protéines, serait le facteur limitant des naines. Cependant, l'augmentation du taux énergétique de la ration de 2,87 à $3,31 \mathrm{k}$.cal. par g. n'avait que peu ou pas d'effet sur la ponte ou la taille des œufs. L'accroissement de 25 p. 100 (par rapport à la ration normale) des quantités de vitamines et des oligo-éléments et l'addition de DL méthionine à la ration des pondeuses, de même, avait un effet faible ou nul sur les performances des poules mises en poulailler.

Afin de tester pourquoi les oiseaux nains ne répondent pas à des niveaux accrus des éléments de la ration, une recherche a été entreprise pour réterminer si la naine était un animal hypothyroïdien. Des poussins commerciaux normaux et des nains ont reçu des rations contenant divers taux de thyroprotéines et de thiouracile. La thyroprotéine avait pour effet une augmentation significative du poids corporel des naines pendant la période de croissance, ainsi qu'un allongement des tarses, aucun effet significatif n'étant noté avec les animaux normaux.

La température corporelle des oiseaux nains a été trouvée d'environ $0,5^{\circ} \mathrm{C}$ plus basse que celle des normaux. L'administration de thyroprotéine a eu pour résultat l'élévation de la température corporelle des nains au niveau de celle des normaux, tandis que le thiouracile produisait un abaissement de la température corporelle des normales et pas de changement chez les naines.

La composition des carcasses indiquait que les naines avaient une proportion de graisse corporelle significativement plus élevée que les normales. La thyroprotéine avait pour effet une diminution de la graisse des carcasses chez les naines, mais une augmentation chez les oiseaux normaux. L'addition de thiouracile aux rations conduisait à un taux de lipides plus élevé dans la carcasse des naines, mais à un taux diminué chez les normales.

Il fut alors décidé d'assurer la croissance de poulettes naines avec une ration normale jusqu'à 20 semaines d'âge, puis essayer d'augmenter leur consommation alimentaire et par suite leur ponte et la taille de leurs cufs par l'adjonction de thyroprotéine dans l'aliment. Différents taux furent essayés avec peu ou pas de succès. Cependant, dans le dernier test, avec des taux aussi faibles que 0,009 p. 100 , les performances des naines apparurent améliorées. D'autres travaux sont nécessaires pour tirer une conclusion définitive dans ce domaine.

Une autre étude a été entreprise avec des rations à 16 et 19 p. 100 de protéines, contenant toutes deux des taux augmentés de vitamines et d'oligo-éléments ainsi qu'un supplément de méthionine. Il n'y a pas eu de différence en réponse aux traitements. Les pontes individuelles étaient enregistrées. La plupart des poules pondaient des séries de deux cufs ou moins. Ces résultats confirment des travaux rapportés antérieurement, suggérant que la taille des séries doit être améliorée avant que l'on puisse obtenir une amélioration majeure de la production.

Dans un autre travail, divers constituants sanguins des naines ont été comparées à ceux de Leghorn blanches normales. Il n'y avait pas de différence significative dans le volume des éléments cellulaires, l'hémoglobine, le nombre d'hématies, ou la concentration moyenne d'hémoglobine corpusculaire. Il en était de même pour le cholestérol total, les protéines ou l'albumine du sérum.

Les acides aminés du plasma ont été déterminés sur des poulets nains et normaux après 8 heures d'alimentation. La plupart ne présentaient pas de différence ; cependant, il y avait une décroissance marquée du taux de méthionine pour les nains comparés aux normaux $(0,78$. contre $6,10 \mu$ mole $/ 100 \mathrm{ml}$ de plasma). D'autres travaux sont en cours pour vérifier ce résultat.

\title{
EFFETS DE LA RESTRICTION ALIMENTAIRE PENDANT LA PÉRIODE DE CROISSANCE SUR LES PERFORMANCES DES REPRODUCTRICES NAINES
}

B. LECLERCQ ET J.C. BLUM

Station de Recherches avicoles, I.N.R.A., 37 - Nouzilly (France)

Six lots de poulettes âgées de 200 jours, issues d'un croisement nain de type "chair ", ont reçu un régime unique de l'éclosion à la maturité sexuelle. Trois régimes ont été utilisés, différant seulement par le taux protéique ; 18,16 ou 14 p. 100. Le taux énergétique était le même dans tous 
les régimes : $2.800 \mathrm{k}$. cal. E.M. par kg. A 5 semaines d'âge, les animaux ont été quantitativement restreints selon deux programmes pré-établis : l'un moins sévère $(25.000 \mathrm{~K}$.cal. par oiseau de 0 à 24 semaines), l'autre plus sévère $(23.000 \mathrm{~K}$.cal.). Chacun de ces niveaux de restriction était appliqué aux trois régimes, conduisant à un total de 6 traitements.

Durant les premières semaines de la période de croissance, le poids vif était comparativement réduit par les faibles taux protéiques. Le niveau de restriction avait une influence de plus en plus prononcée avec l'âge. Néanmoins, l'effet du taux protéique était encore apparent à 20 semaines d'âge.

Les régimes à plus faible taux protéique donnés pendant la croissance retardaient la maturité sexuelle et réduisaient la production d'œufs, le poids des œufs et le nombre de ces derniers pouvant être incubés. Par contraste, le niveau de restriction avait très peu d'effet sur ces caractères.

Un aliment unique (à 15,7 p. 100 de protéines) était distribué pendant la période de ponte. La consommation pendant cette période dépendait du taux protéique de l'aliment reçu pendant les premières semaines de croissance ; plus élevé était le taux protéique durant la période de début de croissance, plus importante la consommation pendant la période de ponte. Ceci restait vrai, mais moins prononcé, après six mois de ponte, lorsque la consommation journalière d'aliment eût été limitée à 120 grammes par poule. Ce phénomène peut être expliqué en majeure partie par les différences de ponte.

Le poids vif à la fin de la période de ponte (67 semaines) était directement proportionnel au taux protéique de la ration de croissance; les animaux ne compensaient pas leur retard de croissance.

La restriction calorique pendant la période de croissance était très bénéfique aux performances de ponte des naines, peut-être parce qu'elle limitait leur tendance prononcée à l'engraissement. Au contraire, une déficience protéique pendant les premières semaines de croissance causait une réduction légère, mais nette, de la production d'œufs.

\section{RATIONS CONCENTRÉES POUR LES PONDEUSES NAINES}

J.H. QUISENBERRY ( $\left.{ }^{1}\right)$

Texas $A$ and $M$ University, Department of Poultry

Sciences, College Station, Texas, 77843, (U.S.A.)

L'introduction récente sur une large échelle d'un gène de nanisme lié au sexe à hérédité simple dans les programmes de test de divers sélectionneurs de souches commerciales a attiré l'attention sur la taille corporelle comme moyen potentiel d'accroitre le rendement alimentaire des volailles. Les résultats de précédentes expériences à l'Université A et $\mathbf{M}$ du Texas ont suggéré que les pondeuses naines ont besoin d'un aliment plus concentré en éléments nutritifs que leurs sœurs non naines. Dans une expérience incluant des oiseaux non nains divisés en trois classes de poids, légers, moyens et lourds, à 20 semaines d'âge, les animaux de petite taille répondaient plus que les lourds à une augmentation de la concentration de leur aliment. Un autre indice de l'importance qu'il y a à nourrir les oiseaux légers différemment de ceux dépassant le seuil minimum de poids de 1300 grammes a été obtenu antérieurement à partir d'expériences comparant les performances d'animaux éclos au printemps ou à l'automne dans un climat chaud, ainsi qu'à partir des premier et second test de performance "gène-environnement " du Texas.

Deux expériences comparant les performances de ponte des animaux nains et non-nains ont été réalisées. Dans la première, deux régimes (16 et $17 \%$ protéines), avec le même rapport calories/protéines et un, deux ou trois oiseaux par cage de $25,4 \times 45,7 \mathrm{~cm}$ ont été comparés.

(') Nous remercions John W. Bradley, Bill H. Doran et John C. Fowler de leur collaboration. 